\title{
ÉTICA, NATURALEZA Y PENSAMIENTO II. ONTOLOGÍA Y NEUROÉTICA A TRAVÉS DE LA FILOSOFÍA DE SPINOZA
}

\author{
Daniel Álvarez Montero \\ Universidad de La Laguna \\ astral.alv@gmail.com
}

\section{RESUMEN}

En el presente artículo, se trazarán algunas líneas generales de la filosofía de Spinoza con objeto de mostrar su adecuación para la integración de las actuales perspectivas en neuroética, y cómo los enunciados de ésta podrían enriquecerse mediante su inclusión en un proyecto ético como el del autor hispanoportugués. Este proceso se desarrollará a partir de una breve caracterización de su ontología que devendrá en un bosquejo de sus posibles beneficios tanto para esta filosofía como para la neuroética.

Palabras Clave: ontología, neuroética, inmanencia.

ETHICS, NATURE AND THOUGHT II.

ONTOLOGY AND NEUROETHICS THROUGH

THE PHILOSOPHY OF SPINOZA

\section{Abstract}

In this article, some general lines about the philosophy of Spinoza will be outlined with the purpose of showing its adaptation to integrate the new perspectives on neuroethics, and how its statements might be enriched by its inclusion in an ethical project such as the one from Spinoza. This will be done from a brief overview of its ontology towards a sketch of its possible benefits both for this philosophy and neuroethics.

KEYwORDs: ontology, neuroethics, immanence.

DOI: http://doi.org/10.25145/j.laguna.2018.42.003

Revista Laguna, 42; julio 2018, pp. 47-58; ISSN: e-2530-8351 


\section{INTRODUCCIÓN}

La filosofía de Spinoza es célebre por su capacidad para integrar distintas disciplinas -idealmente, todos los ámbitos del conocimiento- en un mismo marco ontológico, común e inmanente, que dé cuenta de la Naturaleza como un todo. Así ha sido señalado repetidas veces por diversos autores, entre ellos, verbigracia, Stuart Hampshire o Harold. H. Joachim. Decía el primero, al respecto de la filosofía de este autor en la década de los cincuenta del siglo pasado, que:

To build a bridge to the contemporary interests, and hence to understanding, it seemed necessary in the 1950s to suggest that Spinoza's metaphysics envisaged the possibility of a unified knowledge of the natural order, and that it exhibited the consequences of such unified knowledge in the areas of morality and politics ${ }^{1}$.

Mucho antes había sugerido Joachim, intérprete clásico de la obra del autor hispanoportugués, que el objeto de una filosofía, y esto parece que puede predicarse respecto del objetivo de la filosofía de Spinoza, era el siguiente:

It is the object of philosophy to interpret experience so as to render it intelligible. A philosophy is successful so far as it enables us to 'think' experience, i. e. to take it in as a coherent system, as a whole which is interconnected by an immanent necessity ${ }^{2}$.

Éste es el planteamiento inicial de su obra, cuyo paradigma es la justificación de una ética sobre la base de un modelo ontológico - cosmológico podría decirse también en nuestros días- de la realidad. Este punto de partida no es baladí si lo que se pretende hoy en día es buscar en la naturaleza el fundamento de la moral y preguntarse por las posibilidades éticas de un sujeto que, desde hace ya un tiempo, no es un objeto extraño arrojado a un cosmos distinto de sí mismo, sino parte inextricable de un universo en expansión, formado por su misma materia y energía, y que se explica sin saltos ni rupturas epistemológicas en continuidad con su propio devenir.

En efecto, y siempre desde una perspectiva materialista e inmanente, de la mano del discurso científico y dejando en suspenso los incontables misterios del cosmos - esto es, evitando anticipar respuestas extracientíficas a la miríada de fenómenos aún desconocidos de la naturaleza-, no cabe hoy considerar al ser humano como el crisol de dos realidades contrapuestas, tales como alma y cuerpo, que se reúnen de inescrutable manera en la formación de un individuo de trasfondo místico.

Las actuales perspectivas en neurobiología han fortalecido toda una nueva corriente cuyo tema de estudio no es el planteamiento de la forma y contenidos de una moral desvinculada del ser humano en tanto producto de la naturaleza, como si ésta lo sobrepasase actuando desde otra esfera, sino que se centra en las posibilidades

${ }^{1}$ Hampshire, Stuart [prol.]: Ethics, Penguin Classics, Londres, 1996, p. viI.

2 Joаснім, Harold H. (1901): A Study of the Ethics of Spinoza, Forgotten Books, Londres, 2012, p. 99. 
materiales de la misma desde su fundamento como ser social. No es desconocido que la filosofía de Spinoza es precursora de ciertas premisas de la psicología y la neurociencia contemporáneas, tal como la indefectible relación entre cuerpo y mente y la afección de las pasiones en la forma de ese bucle mente-cuerpo.

Con todo, y al amparo de su fundamentación ontológica, la filosofía de Spinoza trazó una línea ética que recorría toda la naturaleza desde sus mismos fundamentos hasta la forma de gobierno de las sociedades, bajo la premisa de que «la razón no exige nada que sea contrario a la naturaleza», ${ }^{3}$ pues sea lo que sea ésta, constituye una expresión de la infinita Sustancia, concretada en forma de Naturaleza naturada. En el siguiente epígrafe se examinarán las raíces fundamentales de lo que luego devendrá en una ética que podría integrar y traducir en una filosofía completa de la Naturaleza -mayúscula- los nuevos hallazgos del ámbito de la neurobiología o neurociencia.

\section{ONTOLOGÍA FUNDAMENTAL}

La posibilidad de un modelo ético naturalista descansa nada más -y nada menos- que sobre la realidad biológica, tanto corporal como mental, de cada ser humano. Los conceptos de bien y mal, considerados como absolutos, ya fueron negados allá en el siglo XVII por el propio Spinoza, quien arguyó que bueno y malo se predicaba de ciertas cosas según contextos y sujetos, de tal suerte que lo que podía ser bueno para uno en un momento dado podía ser malo para el otro simultáneamente, e incluso ambas consideraciones variar con el tiempo. Podría parecer hoy una afirmación trivial, pero atesora un rechazo férreo a la existencia de ideales de bondad o maldad propios de contextos como aquél en que la obra de Spinoza fue gestada. En resumen: no puede plantearse un modelo ético naturalista transgrediendo los límites inmanentes de la Naturaleza, introduciendo auxilios imaginativos para responder a la multitud de enigmas en torno a ese cosmos dentro de otro cosmos que supone el cerebro humano.

Así, una ética natural, entendiendo por ello una inmanente al propio ser humano, que no requiera para su formulación de la extracción de verdades situadas en algún otro lugar más allá de la propia realidad humana, debe encontrar su suelo en la naturaleza. La pregunta que será abordada en este epígrafe es ¿por qué los cimientos de la filosofía de Spinoza suponen un sustrato adecuado para integrar en el marco de una filosofía de la naturaleza los más recientes hallazgos de la neurobiología que han devenido en la formación de eso que hoy se conoce como neuroética?

Se recorrerá el camino, no obstante, a la inversa: no se partirá de las actuales perspectivas neuroéticas hasta retrotraerse a un posible suelo, sino que se mostrará la adecuación de éste para aquéllas, en aras de un progreso discursivo ascendente que, Madrid, 2006.

3 E, IV, 18, esc. Se seguirá, para las citas a la Ética, la traducción de Vidal Peña en Alianza, 
en cualquier caso, será planteado de manera muy superficial y laxa, dada la brevedad de las siguientes páginas, cuyo único objetivo no es revelar una nueva verdad sobre esta temática cuanto mostrar la viabilidad de este nuevo horizonte ético y científico desde el marco de esta filosofía.

El rechazo al dualismo parece constituir una clave fundamental para una ética de este tipo. En efecto, no se podría trazar una línea continua desde la ética subyacente al modo humano en tanto expresión natural concreta con el resto del orden natural si ésta en algún punto se rompiera, dejando un dudoso enlace a modo de glándula pineal cartesiana que cuestionaría la verdad de sus enunciados. La clave de una ética naturalista es la intersubjetividad externa, consecuencia de la aplicación de cierta premisa fundamental de cientificidad. Ahora bien, que la ética natural maneje una premisa de objetivad -dígase, para no caer en aspiraciones imposibles, la intersubjetividad de la ciencia que radicaría en la accesibilidad a ese conocimiento- no significa que se pueda obtener, al menos de momento, un conocimiento completo de la ingente cantidad de cuestiones alrededor de este tema; menos significa, por tanto, que ante el desconocimiento se adelante una respuesta extracientífica, que la imaginación venga a parchear lo que aún constituye una laguna del saber.

En definitiva: el monismo se muestra como una premisa no negociable para una ética que pretenda fundarse cabalmente en lo natural. Aunque el monismo spinozista ha sido cuestionado alguna vez -en lengua española tal vez con mayor repercusión por Vidal Peña ${ }^{4}$-, aquí se asumirá una lectura común y sencilla del mismo. Lejos de aparatajes teóricos que intenten apostillar ciertos pasajes para sumirlos en la confusión, se mantendrá la pretendida y manifiesta intención del autor, porque aquél sería otro debate e impediría avanzar hacia el objetivo de estas líneas. Llamar a Spinoza monista, con cualesquiera matices que se le puedan o deban apuntar, no parece constituir una ruptura con respecto al planteamiento manifiesto de su sistema, sino todo lo contrario: supone una adecuada exégesis de su modelo filosófico.

Los elementos fundamentales -sólo unos pocos, de interés para este texto- sobre los que esta concepción se erige son los siguientes, y se encuentran en la primera parte de la Éticas:

1) Unicidad de la Sustancia. Dios -Sustancia o Naturaleza, escrita con mayúscula-, es uno y continuo, autocausado, libre, eterno e infinito. Los atributos

${ }^{4}$ Peña, Vidal: «Eternidad y temporalidad 'en' Spinoza 'hacia' Hegel», en Lafuente, María Isabel (ed.): Estudios sobre filosofía moderna y contemporánea. León, Universidad de León, 1984, p. 73.

5 Se esbozará un rápido repaso por ciertas ideas fundamentales, puesto que trazar la gestación, desarrollo e implicaciones de cada una, así como los problemas que distintos autores y contextos les han atribuido, excede la capacidad de estas brevísimas páginas. En ningún momento pretenden ser tomadas como incuestionables o absolutos intachables, pues podría argüirse, y con razón, que si alguno de estos supuestos se tambalease la posterior interpretación también. No obstante, e independientemente de la certeza en torno a los mismos, interesa para el presente estudio su carácter de reglas generales para la constitución de un proyecto ético que estriba sobre la constitución biológica e inmanente de cada ser humano. 
de la Sustancia son «aquello que el entendimiento percibe de la Sustancia como constitutivo de la esencia de la misma» (E, I, 4 def.). Esta aclaración es importante porque aparta totalmente la idea de que la Sustancia sea una suma o agregado de partes, un compuesto de diferentes elementos. Aunque el ser humano percibe la realidad como en una suerte de dos ámbitos -que en Spinoza se expresa mediante la metáfora del paralelismo-, uno relativo al mundo corpóreo, la extensión, y otro relativo al mundo de las ideas e intelecciones, el pensamiento, ambos constituyen la misma Naturaleza y no son elementos separados de la misma unidos por alguna extraña conexión. Por eso el paralelismo: dos líneas paralelas jamás entrarán en contacto entre sí, en Spinoza ello se justifica porque una se superpone a la otra o, mejor dicho, porque ambas son la misma cosa, pero percibida de dos formas distintas.

2) La Sustancia, en su devenir naturante, se natura-concreta- en forma de modos. Esto es, existe una doble concepción de la Naturaleza: se la considerará naturante cuando se refiera a su infinitiva potencia generativa, como fuerza absolutamente indeterminada; por otro lado, será llamada naturada cuando se refiera a cualquiera de sus productos, finitos o infinitos, en la medida en que son concreciones de aquel poder generativo subyacente a Dios. Todo cuanto es en Dios, y sin Dios nada puede ser ni concebirse (E, I, 15).

3) De lo anterior se desprende que la posición del ser humano en el mundo, tanto en cuerpo como en mente, es la de un modo, o lo que es lo mismo, una manifestación, producto, forma concreta que la Naturaleza adquiere en su infinito devenir, actualización de una esencia en ella contenida. Los modos no son productos aislados de la Sustancia, son ella misma en una configuración -actualización- determinada.

4) Finalmente, y como conclusión de todo lo anterior, Spinoza enuncia que existe sólo una Sustancia y que, por supuesto, al ser del hombre no pertenece el ser Sustancia, sino el modo. A modo de colofón, enuncia algo que supone un suelo fundamental para postular una ética monista, la realidad inmanente de la Naturaleza, al afirmar aquello de que «Dios es causa inmanente, pero no transitiva, de todas las cosas» $(\mathrm{E}, \mathrm{I}, 18)$.

Manejando estos puntos, que en este texto se alzarán como simples postulados y cuya justificación y problemas aducidos a lo largo del tiempo deben ser obviados por cuestiones de espacio, podrá arrostrarse la ulterior exposición respecto de una aspiración ética sobre ellos fundada, a modo de principios generales y no de verdades absolutas.

\section{CONATUS: EL AVANCE DESDE LA ONTOLOGÍA A LA ÉTICA}

Lo fundamental en la filosofía de Spinoza, motivo por el que ha sido reivindicada aquí a propósito de este discurso, es que su proyecto ético aparece como consecuencia de una ontología, y aunque el tema central es siempre una ética para el ser humano -no en vano, su gran obra se llamó Ética-, ésta no pretende surgir 
de la nada, sino enraizar en la misma esencia de la Naturaleza. Es decir, Spinoza no defiende un modelo ético porque le parece el más útil para manejar al ser humano o sus sociedades, sino que lo extrae de lo que, a su juicio, se deriva consecuentemente de la propia esencia de la Naturaleza. Esta pretensión, con independencia de si resulta plausible o no, es a lo que aspira una ética que quiera preguntarse qué hay en la propia naturaleza de la especie para proclamar o reivindicar un comportamiento sobre la base de aquello que es considerado mejor o peor o, en términos de su filosofía, capaz de aumentar o reducir la potencia natural.

El valor de este sesgo marcado a través del modelo de Spinoza debe ya resultar claro: todo lo que pretenda ser una ética genuinamente naturalista debe siempre mantenerse sobre esa línea, la de promover un discurso que, en todo momento, camine de la mano de unos enunciados que tomen como sustrato la cada vez más conocida naturaleza humana -siendo aún un ignoto océano por cartografiar-. Por supuesto, es imposible hoy, y tal vez lo sea siempre, desvelar todos los secretos de esa suerte de cosmos que supone un cerebro humano, pero su desconocimiento no es óbice para reconocer que parece haber un camino inmanente desde su constitución a su traducción en la forma de pensamientos, ideas y conductas, donde las nuevas investigaciones en neurobiología tienen cada vez más pistas sobre su realidad.

En el siglo XVII, Spinoza supo ver una identificación entre los sucesos del cuerpo y los de la mente, y aunque no existiese una ciencia tan desarrollada como la actual, ya le fue posible entonces entender que las pasiones y acciones humanas venían predispuestas por su propia naturaleza, y no la excedían de ninguna manera. En efecto, la razón no pedía nada contra la Naturaleza, y el prólogo de la segunda parte de la Ética ya suponía toda una declaración de principios, al enunciar que pasaba a explicar, para el conocimiento de la mente humana y su suprema felicidad, «aquellas cosas que han debido seguirse necesariamente de la esencia de Dios, o sea, del Ser eterno e infinito» (E, II, prol.).

Ni las virtudes ni las perversiones humanas provenían de ningún ámbito oscuro más allá de la Sustancia o, lo que es lo mismo, la Naturaleza ${ }^{6}$, una controvertida conclusión difícilmente encajable en su contexto. ¿Qué implicaba realmente? ¿Acaso la inconsistencia humana, sus mayores errores y oscuridades, provenían también de esa Sustancia spinozista? Por sorprendente que pareciera en su época, era eso lo que implicaba, pero porque ese Dios de Spinoza mucho distaba de aquella instancia divina superior con voluntad para obrar y decidir en cada momento como si de un juez de corte antropomórfico y omnipotente se tratase.

En efecto, Deus sive Natura no es otra cosa que desproveer a Dios de esa imagen en la que parece un ser humano más, que aplica su voluntad aquí o allá, bajo un halo de omnipotencia y bondad absolutas. El Dios spinozista contiene todas las peculiaridades de la Naturaleza, pero no la jerarquiza en ningún momento. Ni la personalidad, ni la voluntad, etcétera, serán en ningún caso su nota genérica, sino sive Natura.

${ }^{6}$ No debe nunca ignorarse aquella equiparación spinozista entre Dios y Naturaleza: Deus 
concreciones, posibilidades suyas, de su infinito devenir y potencia generativa. Todo lo bueno y lo malo, toda la obra de cada ser humano, está contenida en sí mismo y en relación con su medio, de ella debe partir hasta donde alcance su limitada potencia, y es tan divina -en el sentido de producida por la Sustancia- como cualquier otro elemento del cosmos.

Se entronca aquí la inveterada cuestión en torno al panteísmo spinozista, que parece ser más una discusión espuria de nombres que algo relevante de cara a su contenido. Si Dios es igual a Naturaleza, ¿todo es Dios? Sí, en un sentido spinozista. No obstante, cada ser humano es sólo una concreción de esa Naturaleza infinita, no la agota ni muchísimo menos. Ningún elemento naturado es totalmente Dios, pues contravendría su noción de infinitud e indeterminación absolutas; pero, sin embargo, ninguno de los modos es algo distinto a Dios, ni en sus formas pensantes y en apariencia más sofisticadas, ni en las más simples formas de materia.

Esta perspectiva filosófica deja al ser humano como un modo en continua relación, como si de una física de las pasiones y los pensamientos se tratase, con esa Naturaleza que le dio origen y que marcará también el límite de su estructura modal individuada. A tenor de esta exposición, no puede concebirse el proyecto ético spinozista como algo desvinculado de esta realidad y su indefectible inmanencia: lo que quiera que sea una actuación humana lo será brotando a partir de esta raíz naturalizada, y sólo desde ella retoñará en una ética.

El paso desde esta ontología spinozista hacia un horizonte ético sería inviable sin una noción fundamental de esta filosofía, el conatus: "Cada cosa se esfuerza, cuanto está a su alcance, por perseverar en su ser» (E, III, 6). Mucho se ha escrito sobre éste, y no parece haber consenso al respecto de sus consecuencias e implicaciones para la filosofía de Spinoza. Algunos autores otorgan un sentido vitalista al conatus -más o menos explícito-, pero también podría proponerse que es más un principio ontológico que algo que se manifieste a través del existir biológico y consciente humano. El presente discurso se mantendrá sobre esta última interpretación, pues parece la única que permite esquivar sin grandes dificultades toda la problemática aducida a este concepto.

Apartando, pues, la controversia en torno a de qué manera el conatus podría manifestarse en el caso de los seres humanos, puede situarse su origen como consecuencia de la cuarta proposición de la tercera parte de la Ética, que reza: «Ninguna cosa puede ser destruida sino por una causa exterior». En un sentido ontológico, fundamental, lo que constata este concepto es la tendencia al ser de la Naturaleza, en oposición a la nada. No quiere decir, desde luego, que la destrucción no sea posible dentro de ella. En un sentido muy laxo, el conatus, a través de cada modo, supone la perpetuación de aquel principio fundamental de la Sustancia spinozista que le otorgaba existencia necesaria: «A la naturaleza de una substancia pertenece el existir» (E, I, 7).

El conatus, pues, es una consecuencia de la propia realidad ontológica de la Sustancia y, por tanto, en todas y cada una de las concreciones de la misma este principio se manifiesta -si desapareciera se habría producido una ruptura con el orden inmanente de la misma-, lo que no evita que algunos de estos modos hayan devenido inestables dentro de la configuración modal de la que forman parte y puedan desintegrarse o aniquilarse, sin que ello contravenga aquella cuarta 
proposición de la tercera parte. La pregunta que interesa recuperar aquí es ¿̇tiene traducción mental esta noción en términos humanos? Es problemático darle un sentido psicológico más que en forma de reflexión acerca de este concepto, pues entonces sería difícil conciliar esa cuarta proposición de la tercera parte de la Etica con el caso del suicidio7. Psicologizar el conatus hasta el punto de asumir que existe en la obra de Spinoza una imposibilidad racional para un ser vivo de aniquilarse a sí mismo supone una interpretación abierta a una inveterada problemática aparentemente ajena a cualquier consenso. En definitiva, puede decirse que este concepto persiste a través del modo humano ontológicamente, pero proponerlo biológica o psicológicamente más que en la forma de una reflexión sobre la realidad ontológica del conatus abre una problemática compleja. Parece pertinente esta breve digresión acerca de las dificultades de ese tipo de conatus porque abriría la proyección de esta ética naturalista a terrenos exageradamente especulativos, cuando no la fundaría sobre un suelo inconsistente y carente de solidez.

\section{Cerebro y mundo, LA imposibilidad del Aislamiento}

Parece claro que no hay cerebros al margen de sus circunstancias. Tanto en su formación como en su desarrollo ulterior, el cerebro humano está en continua relación con procesos externos cruciales para su propio funcionamiento. Es cierto, se dirá, que la conciencia también se objetiva a sí misma, con cierta transparencia -no completa, a razón de todo cuanto se ha descubierto en torno al inconsciente y los procesos mentales inaccesibles-, pero que la formación de un cerebro requiere de su relación con un mundo, donde además el cuerpo a través de los sentidos provee de información a aquél, parece una realidad inherente a cada ser humano.

Podría recuperarse a este respecto el concepto de exocerebro de Bartra, que encaja de manera excelente con la ontología spinozista. Señalando el carácter utópico de aquel hombre en estado de naturaleza, Bartra aduce igualmente la misma problemática en torno a las consideraciones en abstracto del cerebro:

Sabemos desde hace mucho tiempo que el hombre en estado de naturaleza no existió más que en la imaginación de los filósofos y naturalistas ilustrados. Y podemos sospechar que el hombre neuronal desnudo tampoco existe: un cerebro humano en estado de naturaleza es una ficción ${ }^{8}$.

7 Diana Cohen ha tratado y considerado el problema del conatus detalladamente desde esta perspectiva. Su trabajo merece una mención especial: $c f$. CoHen, Diana: El suicidio: deseo imposible o la paradoja de la muerte voluntaria en Baruj Spinoza, Ediciones del signo, Buenos Aires, 2003. También de la misma autora: Acerca de la posibilidad de un imposible: el suicidio en la lente de Spinoza, en Fernández, Eugenio y La Cámara, María Luisa de (eds.): El gobierno de los afectos en Baruj Spinoza, Trotta, Madrid, 2007, pp. 483-495.

${ }^{8}$ Bartra, Roger: La conciencia y los sistemas simbólicos, pre-textos, Valencia, 2006, p. 20. 
La sintonía con la obra de Spinoza, a nivel ontológico, es notoria aquí. En efecto, el hispanoportugués había negado todo carácter de Sustancia al ser humano, en lo corpóreo y en lo mental, y había escogido el término mens en vez de anima para referirse a esa faceta intelectiva del ser humano. En su condición de modo, el hombre forma parte de una inextricable y complejísima red modal que lo origina y lo determina. El cerebro no puede, ni al amparo de la obra de Spinoza, ni de acuerdo al sentido común de las nuevas indagaciones neurocientíficas, operar en abstracto, aislado de la realidad de la que se nutre y que lo moldea. Afirmaba también Bartra:

Mi hipótesis supone que ciertas regiones del cerebro humano adquieren genéticamente una dependencia neurofisiológica del sistema simbólico de sustitución. Este sistema, obviamente, se transmite por mecanismos culturales y sociales. Es como si el cerebro necesitase la energía de circuitos externos para sintetizar y degradar sustancias simbólicas e imaginarias, en un peculiar proceso anabólico y catabólico?

Por supuesto, el concepto de Bartra y su alcance va más allá de la profundidad de estas líneas, pero conviene recuperar esa noción de unión, de pertenencia, de concatenación entre el cerebro y lo externo, sea lo cultural, lo social, lo político, lo físico o lo ontológico. En definitiva, una ética naturalista tiene sus limitaciones, un sendero del que no puede separarse, o su pretensión de intersubjetividad, de corroboración científica, se esfumaría irremediablemente. El proyecto ético al que estas páginas apuntan tendrá esa limitación, y acaso será ése su mayor privilegio: moverse estrictamente en lo que, de manera global para todo ser humano, supone un conjunto de enunciados razonables y abarcables desde premisas revisables, refutables y contrastables.

\section{ÉTICA Y NEUROÉTICA}

La neuroética es una disciplina derivada tanto del avance de las neurociencias como, por supuesto, de la filosofía en cuanto a la repercusión y alcance de sus temas. El descubrimiento cada vez mayor de las distintas zonas cerebrales y su funcionamiento, así como algunas de sus posibles repercusiones no ya para la psique humana, sino en su vertiente social o exterior, en la manera en que los seres humanos determinan una sociedad sobre la base de esta biología en proceso de descubrimiento, ha mostrado ser no un aspecto anecdótico en las nuevas investigaciones tanto en neurociencias como en filosofía, sino una necesidad surgida por el alcance de estos nuevos enunciados.

Expresado de forma sencilla: parece pertinente, para una reflexión en torno a la moral o la ética, e incluso para el alcance político de esos enunciados, tomar en consideración lo que estas nuevas disciplinas acerca del cerebro humano revelan del sujeto ético sobre el que se pretende teorizar. Incluso en el siglo XVII, cuando aún

9 Ibidem, p. 26. 
quedaba lejos este horizonte, la filosofía de Spinoza, con su proyección del bucle mente-cuerpo, parecía aspirar a una reflexión de este tipo que pudiera incluir en sus fundamentos un suelo biológico sobre el que erigir su ética. No en vano, la figura de Spinoza ha adquirido en la contemporaneidad un reconocimiento que no alcanzó en su tiempo, pues resultaba difícil de encajar en aquel contexto, y su recuperación en la actualidad es manifiesta incluso más allá de la filosofía ${ }^{10}$.

La filosofía de Spinoza, aun con esta distancia respecto del auge de las neurociencias, resulta ser una exégesis excelente acerca de la pertinencia de la integración de la neuroética en un marco filosófico como el suyo. En efecto, y de nuevo en sintonía con las palabras de Hampshire, en la medida en que el modelo spinozista promueve ese conocimiento unificado del orden natural, no sólo no se opone, sino que aspiraría a que las nuevas investigaciones contribuyan a la solidificación de ese suelo que, si bien en el siglo Xvir tenía un aspecto más teórico, hoy puede alcanzar un cariz más empírico. En otras palabras, no parece propio de la filosofía de Spinoza ignorar lo que el discurso científico va revelando del mundo, sino, muy al contrario, utilizar ese conocimiento para orientar un proyecto ético.

En palabras de António Damásio:

Spinoza es relevante para la neurobiología a pesar del hecho de que sus reflexiones sobre la mente humana surgieran de una preocupación a mayor escala por la condición de los seres humanos [...]. Su pensamiento desciende del de Aristóteles, pero el fundamento biológico es más firme, lo cual no es ninguna sorpresa. Parece que Spinoza entresacó una relación entre la felicidad personal y colectiva, por un lado, y la salvación humana y la estructura del Estado, por otro, mucho antes de que lo hiciera John Stuart Mill ${ }^{11}$.

Es notorio que la obra de Spinoza promueve esa relación bidireccional entre el ser humano y el mundo. La mente contribuye a la determinación del medio, y éste a su vez afecta a la mente. Este medio, externo, no se identifica únicamente con el mundo en sentido físico o corpóreo, sino también con la estructura social y política inherente a las colectividades humanas. Con las cada vez más numerosas indagaciones sobre la estructura del cerebro humano, aquella caja negra que parecía accesible casi exclusivamente mediante la introspección ha empezado a ser objetivada, estudiada externamente, en una investigación que ha propiciado hallazgos imposibles de dejar al margen de una perspectiva moral.

Un ejemplo lo constituye el caso de las neuronas espejo, cuya capacidad especular parece desempeñar una importante labor en esta relación con el resto de individuos y, por extensión, con el desarrollo de un comportamiento social. Este fun-

${ }_{10}$ Dentro de la filosofía, por ejemplo, la obra de Hernández Pedrero, Vicente: Ética de la inmanencia: el factor Spinoza, Servicio de publicaciones ULL, La Laguna, 2011. Del lado de la neurociencia, el célebre trabajo En busca de Spinoza de DAmásio, António: Looking for Spinoza: Joy, Sorrow, and the Feeling Brain, Harcourt, Orlando, 2003.

11 Damásio, António: En busca de Spinoza, Crítica, Barcelona, 2009, p. 21. 
damento biológico supone una conquista que ha propiciado una mayor comprensión de la realidad mental y no parece pertinente ignorarlo de cara al estudio inmanente de una ética. Inmanencia sigue siendo, aquí, concepto fundamental para el desarrollo de una neuroética, esto es, una ética que no sólo se mueve en la introspección reflexiva acerca de lo bueno y lo malo, lo que aumenta o resta potencia, sino que busca en su propia constitución biológica los fundamentos de esa inclinación a la vida.

Si el conocimiento acerca de lo bueno y lo malo, de lo que elevaría al ser humano a un mejor estado de salud, adquiere ahora nueva significación sobre la base del descubrimiento de mecanismos cerebrales que ayudan a comprender de dónde brota esa comprensión del sí mismo y del otro, así como de lo que causa alegría y de lo que no, entonces parece innegable que este contenido se integra inextricablemente en una disciplina que tenga por objeto cuestionar y evaluar el comportamiento humano en relación con el bien, el mal, la felicidad o la realización personal.

\section{SPINOZISMO Y NEUROÉTICA}

No sólo de neuroética, sino también de neuropolítica, se ha venido hablando en los últimos ańos. Se trazaron, primero, unas breves líneas muy generales sobre la ontología spinozista, así como unos apuntes sobre la naturaleza del conatus y su posible problemática y, finalmente, unas líneas generales sobre la aceptada relación entre el cerebro y el mundo y la relevancia de una disciplina incipiente como la neuroética en el estudio del comportamiento humano. Es cierto que la obra magna de Spinoza se llamó Ética por algo: su razón es un proyecto ético. Las características e implicaciones de ese proyecto constituyen lo que podría ser de interés para incluir los actuales avances en neurociencia en una filosofía como ésta.

Tal vez el primer motivo, que ya se ha dejado entrever, responde a esa ausencia de hermeticidad en la filosofía de Spinoza. Dicho de otro modo, la filosofía de Spinoza no se presenta como un bloque definitivo y acabado, sino que está abierta a todo cuanto un marco científico pueda otorgarle ${ }^{12}$. Este aspecto no es baladí, pues, aunque el proyecto ético de Spinoza se deja ver en sus líneas generales en la Ética, el tiempo ha mostrado que el contenido de sus enunciados puede $-y$, para una revaloración en el presente, debería- ser actualizado y reformulado con los nuevos avances, con objeto de ampliar su discurso con un nivel de rigor que no sólo complementaría esta filosofía, sino que la reforzaría con un suelo científico más estable sobre el que desplegar sus enunciados.

La Ética de Spinoza apunta a una salvación, que aparece nombrada como beatitudo, y que podría traducirse por felicidad suprema, donde suprema incluye necesariamente la libertad: «Un hombre libre en nada piensa menos que en la muerte,

${ }^{12}$ Harold Joachim sugería, por ejemplo, que, de haber sido completado el proyecto spinozista, éste incluiría trabajos como teorías de la mecánica, sobre moral o una medicina para el cuerpo, entre otros. Jоаснім, Harold H.: op. cit., n. ${ }^{\circ} 2$, p. 4. 
y su sabiduría no es una meditación de la muerte, sino de la vida» (E, IV, 67). En efecto, la felicidad spinozista implica una superación de los miedos y prejuicios humanos, es emancipadora en virtud del desarrollo de la potencia de acción de cada ser humano. "La felicidad no es un premio que se otorga a la virtud, sino que es la virtud misma...» $(\mathrm{E}, \mathrm{V}, 42)$.

Si a nivel ontológico la naturaleza muestra su realidad necesariamente existente, a nivel biológico es manifiesto que los seres vivos propenden a una continuación en su ser, desarrollando su potencia de acción en la medida en que les es posible. La Ética de Spinoza es una ética de y para la vida, que la promueve y la potencia, que se funda en la alegría y que es emancipadora precisamente en cuanto su sabiduría es, como se dijo, meditación sobre la propia vida.

La investigación neurocientífica en torno a los mecanismos cerebrales que sustentan el comportamiento, que tienden al desarrollo y la conservación de la vida, suponen un suelo biológico, empírico, a aquella reflexión ética hacia la que apunta la obra de Spinoza. La integración de estos enunciados neurocientíficos en el marco spinozista los traduce a un lenguaje que trasciende la mera constatación fáctica o experimental y los convierte en fundamento ético, razón de aquella aspiración a la beatitudo, una salvación adquirible en términos inmanentes.

En este sentido, la predisposición biológica a la autoconservación, presente igualmente en la filosofía spinozista, puede expresarse en ella en un lenguaje más allá del científico que permite, por ejemplo, hablar de potencia humana, y de su ampliación o reducción en términos inmanentes y hacia una mejora del estado de salud psicosomático del individuo, que avanza desde estos enunciados fundamentales hacia esa salvación en términos de felicidad suprema y que, además, aspira a traducirse en un discurso político, lo que muestra la inextricable unión, dentro de ese marco inmanente, de una ontología con una biología y, a partir de ella, con una ética y una política.

En resumen, la filosofía spinozista promueve una praxis ética, un desarrollo vital fundado sobre estos principios que ahora las neurociencias pueden comenzar a esbozar y que suponen el sustrato naturalista, inmanente y monista de una ética que no pide nada contra la naturaleza, y que en ella misma ancla sus razones y aspiraciones. Esa beatitudo no supone ningún tipo de anhelo místico más allá de los límites de la naturaleza, sino que se plantea como una salvación en sus propios términos, por ello brota de sus cimientos y desde sus raíces se despliega sub specie aeternitatis. Desde una perspectiva conjunta, la inclusión de las actuales investigaciones neurocientíficas en la filosofía de Spinoza podría, pues, dar un sustrato empírico a este modelo de una manera que no era posible en el siglo XVII, y permitiría a estos hallazgos científicos formar parte de un marco inmanente que extendería el alcance de sus enunciados, en la medida en que formarían parte de un proyecto ético que apunta hacia esa felicidad suprema y que se funda en una ontología como la descrita. 